\title{
Increasing subsequences and the hard-to-soft edge transition in matrix ensembles
}

\author{
Alexei Borodin ${ }^{1}$ and Peter J Forrester ${ }^{2}$ \\ ${ }^{1}$ School of Mathematics, Institute of Advanced Study, Einstein Drive, Princeton, NJ 08540, USA \\ 2 Department of Mathematics and Statistics, University of Melbourne, Victoria 3010, Australia \\ E-mail: borodine@math.upenn.edu and p.forrester@ms.unimelb.edu.au
}

Received 28 August 2002, in final form 17 December 2002

Published 12 March 2003

Online at stacks.iop.org/JPhysA/36/2963

\begin{abstract}
Our interest is in the cumulative probabilities $\operatorname{Pr}(L(t) \leqslant l)$ for the maximum length of increasing subsequences in Poissonized ensembles of random permutations, random fixed point free involutions and reversed random fixed point free involutions. It is shown that these probabilities are equal to the hard edge gap probability for matrix ensembles with unitary, orthogonal and symplectic symmetry respectively. The gap probabilities can be written as a sum over correlations for certain determinantal point processes. From these expressions a proof can be given that the limiting form of $\operatorname{Pr}(L(t) \leqslant l)$ in the three cases is equal to the soft edge gap probability for matrix ensembles with unitary, orthogonal and symplectic symmetry respectively, thereby reclaiming theorems due to Baik-Deift-Johansson and Baik-Rains.
\end{abstract}

PACS numbers: $02.10 . E b, 05.40 .+\mathrm{j}$

\section{Introduction}

Let $S_{N}$ denote the set of all permutations of $\{1,2, \ldots, N\}$. Let $\pi \in S_{N}$ and consider a subsequence of image points $\left\{\pi\left(i_{1}\right), \pi\left(i_{2}\right), \ldots, \pi\left(i_{k}\right)\right\}$ where $1 \leqslant i_{1}<\cdots<i_{k} \leqslant N$. Such a subsequence is referred to as an increasing subsequence of length $k$ if $\pi\left(i_{1}\right)<\pi\left(i_{2}\right)<$ $\cdots<\pi\left(i_{k}\right)$. For a given $\pi$, let $L_{N}(\pi)$ denote the maximum length of all the increasing subsequences. The question of the distribution of $L_{N}(\pi)=: L_{N}$, when $\pi$ is chosen at random from a uniform distribution on $S_{N}$, was posed in the early 1960s by Ulam. In 1999 the question was answered by Baik, Deift and Johansson [3], who proved

$$
\lim _{N \rightarrow \infty} \operatorname{Pr}\left(\frac{L_{N}-2 \sqrt{N}}{N^{1 / 6}} \leqslant s\right)=F_{2}(s)
$$

where $F_{2}(s)$ is the scaled cumulative distribution of the largest eigenvalues for large random Hermitian matrices with complex Gaussian entries (technically matrices from the Gaussian 
unitary ensemble (GUE)) [35]. One should consult [2] for a review of the work on Ulam's problem culminating in the Baik-Deift-Johansson theorem.

In the course of proving (1.1), the exponential generating function of $\operatorname{Pr}\left(L_{N} \leqslant l\right)$,

$$
\mathrm{e}^{-t} D_{l}(t) \quad D_{l}(t):=\sum_{N=0}^{\infty} \frac{t^{N}}{N !} \operatorname{Pr}\left(L_{N} \leqslant l\right)
$$

was introduced. This quantity itself is the cumulative distribution of a natural quantity due to Hammersley (see, e.g., [1]). Thus consider the unit square with points chosen at random according to a Poisson process of rate $t$. Form a continuous piecewise linear path, with positive slope where defined, connecting $(0,0)$ to $(1,1)$ and only changing slope at a point. Let $L^{\square}(t)$ denote the length of the longest such 'up/right' path, where the length is defined as the number of Poisson points in the path. To see the relation to (1.2), label the points $1, \ldots, N$ from left to right, then attach a second label $1, \ldots, N$ from bottom to top. In this way each array of $N$ points is associated with a permutation, and furthermore the fact that the points are chosen from a Poisson process implies the uniform disitribution on the set of permutations of $N$ symbols. Up/right paths correspond to increasing subsequences and we have

$$
\operatorname{Pr}\left(L^{\square}(t) \leqslant l\right)=\mathrm{e}^{-t} D_{l}(t) .
$$

It was proved in [3] that

$$
\lim _{t \rightarrow \infty} \operatorname{Pr}\left(\frac{L^{\square}(t)-2 \sqrt{t}}{t^{1 / 6}} \leqslant s\right)=F_{2}(s) .
$$

In fact (1.4) suffices to prove (1.1), by applying a so-called de-Poissonization lemma [19].

Four companion identities to (1.4), relating the limiting distribution of longest paths in certain up/right path problems to the limiting distribution of the largest eigenvalue in certain random matrix ensembles, were found by Baik and Rains [4, 5]. Of these two are independent, in that it was shown that the other two follow as corollaries [4, theorem 2.5]. For the first, modify the original longest up/right path problem by requiring that initially only the region below the line $y=1-x$ of the unit square be filled with Poisson points of rate $t$; the points above the line are then specified by the image of the initial points reflected about $y=1-x$. Let $L^{\square}(t)$ refer to the longest up/right path from $(0,0)$ to $(1,1)$ in this setting. Then, one has

$$
\lim _{t \rightarrow \infty} \operatorname{Pr}\left(\frac{L^{\square}(t)-2 \sqrt{t}}{t^{1 / 6}} \leqslant s\right)=F_{1}(s)
$$

where $F_{1}(s)$ is the cumulative distribution of the largest eigenvalue for large random real symmetric matrices with Gaussian entries (technically matrices from the Gaussian orthogonal ensemble (GOE)) [37]. The random variable $L^{\square}(t)$ is related to the maximum length $L_{2 N}^{\square}$ of all decreasing subsequences of random fixed point free involutions $\left(\pi^{2}=\pi, \pi(i) \neq i\right.$ for any $i$ ) of $\{1,2, \ldots, 2 N\}$, or equivalently of all increasing subsequences of reversed fixed point free involutions. Thus,

$\operatorname{Pr}\left(L^{\square}(t) \leqslant l\right)=\mathrm{e}^{-t / 2} D_{l}^{\square}(t) \quad D_{l}^{\square}(t):=\sum_{N=0}^{\infty} \frac{t^{N}}{2^{N}} \frac{\operatorname{Pr}\left(L_{2 N}^{\square} \leqslant l\right)}{(2 N) !} \quad(l>0)$.

For the second of the companion identities, the original longest up/right path problem is modified by requiring that initially only the region below the line $y=x$ of the unit square be filled with Poisson points at rate $t$, with the points above the diagonal specified as the image of these points reflected about $y=x$. With $L^{\square}(t)$ referring to the longest up/right path in this 
setting, one has

$$
\lim _{t \rightarrow \infty} \operatorname{Pr}\left(\frac{L^{\square}(t)-2 \sqrt{t}}{t^{1 / 6}} \leqslant s\right)=F_{4}(s)
$$

where $F_{4}(s)$ is the scaled cumulative distribution of the largest eigenvalue for large random Hermitian matrices with real quaternion elements (technically matrices from the Gaussian symplectic ensemble (GSE)). With $L_{2 N}^{\square}$ denoting the maximum length of increasing subsequences of random fixed point free involutions of $\{1,2, \ldots, 2 N\}$, one has

$\operatorname{Pr}\left(L^{\square}(t) \leqslant l\right)=\mathrm{e}^{-t / 2} D_{l}^{\square}(t) \quad D_{l}^{\square}(t):=\sum_{N=0}^{\infty} \frac{t^{N}}{2^{N}} \frac{\operatorname{Pr}\left(L_{2 N}^{\square} \leqslant l\right)}{(2 N) !} \quad(l>0)$.

In this paper we will give new proofs of the results (1.4), (1.5) and (1.7). The original proof of (1.4) uses a Riemann-Hilbert analysis [3]. The subsequent proofs of (1.4) given in $[7,21]$ rely on proving the convergence of a certain Fredholm integral operator determing $\operatorname{Pr}\left(L^{\square}(t) \leqslant l\right)$ to the Fredholm integral operator determining $F_{2}(s)$. A combinatorial proof exploiting the interplay between maps and ramified coverings of the sphere is given in [27]. In the cases of (1.5) and (1.7), a Riemann-Hilbert analysis was again used in the original proof [5]. No other derivations of (1.5) and (1.7) have previously been given. Our derivation relies on finding expressions for $\operatorname{Pr}\left(L^{\square}(t) \leqslant l\right), \operatorname{Pr}\left(L^{\square}(t) \leqslant l\right)$ and $\operatorname{Pr}\left(L^{\square}(t) \leqslant l\right)$ as sums over correlations determining the probability of the interval $(0, t)$ being eigenvalue free in the infinite, scaled Laguerre unitary ensemble (LUE), Laguerre orthogonal ensemble (LOE) and Laguerre symplectic ensemble (LSE) with parameter value $a=l$. The latter are known as hard edge gap probabilities. The probabilities $F_{2}(s), F_{1}(s)$ and $F_{4}(s)$ give the so-called soft edge gap probability that the interval $(s, \infty)$ is eigenvalue free in the infinite scaled GUE, GOE and GSE respectively, and can also be written as a sum over correlations. The limit formulae (1.4), (1.5) and (1.7) are then proved by establishing the convergence as $a \rightarrow \infty$ of the sum over correlations determining the hard edge gap probabilities, to the sum over correlations determining the soft edge gap probabilities. Related studies of the convergence of the finite $N$ soft edge gap probability in the LOE and LUE to the corresponding scaled soft edge gap probability have previously been undertaken in [20, 22, 33], and it is the method of [33] which we adopt here.

In section 2 we present formulae from the theory of zonal polynomials which allow $\operatorname{Pr}\left(L^{\square}(t) \leqslant l\right), \operatorname{Pr}\left(L^{\square}(t) \leqslant 2 l\right)$ and $\operatorname{Pr}\left(L^{\square}(t) \leqslant 2 l\right)$ to be expressed as hard edge gap probabilities. In section 3 we show how the hard edge gap probabilities can be written as sums over correlations for certain determinantal point processes, and this exercise is repeated for the soft edge gap probabilities $F_{1}(s), F_{2}(s)$ and $F_{4}(s)$. The convergence of the sum over correlations determining the hard edge gap probabilities, to the sum over correlations determining the soft edge gap probabilities, is established in section 4.

\section{Averages over classical groups and the hard edge gap probability in the Laguerre ensemble}

It has been shown by Rains [30] that the generating functions of interest each can be written as averages over classical groups. Thus

$$
\begin{aligned}
& D_{l}(t)=\left\langle\mathrm{e}^{\sqrt{t} \operatorname{Tr}\left(U+U^{\dagger}\right)}\right\rangle_{U \in U(l)} \\
& D_{2 l}^{\square}(t)=\left\langle\mathrm{e}^{\sqrt{t} \operatorname{Tr} S}\right\rangle_{S \in S p(l)} \\
& D_{2 l}^{\square}(t)=\left\langle\mathrm{e}^{\sqrt{t} \operatorname{Tr} O}\right\rangle_{O \in O(2 l)}
\end{aligned}
$$


(we use the notation $\operatorname{Sp}(l)$ to denote $l \times l$ unitary matrices with real quaternions elements, or equivalently $2 l \times 2 l$ symplectic unitary matrices with complex elements).

The average (2.1) earlier appeared as the cumulative distribution of the smallest eigenvalue for the scaled LUE [10]. We recall the LUE refers to the eigenvalue probability density function

$$
\frac{1}{C} \prod_{l=1}^{N} \lambda_{l}^{a} \mathrm{e}^{-\lambda_{l}} \prod_{1 \leqslant j<k \leqslant N}\left(\lambda_{k}-\lambda_{j}\right)^{2} \quad \lambda_{l}>0
$$

For $a=n-N, n \geqslant N$, it is realized by eigenvalues of the matrix $X^{\dagger} X$, where $X$ is an $n \times N$ complex Gaussian matrix. The cumulative distribution of the smallest eigenvalue for the ensemble (2.4) (or what is the same thing, the probability of no eigenvalues in the interval $(0, s))$, to be denoted by $E_{2}^{L}(s ; a ; N)$, is obtained from $(2.4)$ by integrating each of the eigenvalues over $(s, \infty)$,

$E_{2}^{L}(s ; a ; N):=\frac{1}{C} \int_{s}^{\infty} \mathrm{d} \lambda_{1} \lambda_{1}^{a} \mathrm{e}^{-\lambda_{1}} \cdots \int_{s}^{\infty} \mathrm{d} \lambda_{N} \lambda_{N}^{a} \mathrm{e}^{-\lambda_{N}} \prod_{1 \leqslant j<k \leqslant N}\left(\lambda_{k}-\lambda_{j}\right)^{2}$

(the normalization $C$ is such that $\left.E_{2}^{L}(0 ; a ; N)=1\right)$. Rescaling the eigenvalues in the vicinity of the origin (referred to as the hard edge since the eigenvalues are restricted to $\lambda_{l}>0$ ) by

$$
\lambda_{l} \mapsto \frac{x_{l}}{4 N}
$$

the correlations have a well-defined $N \rightarrow \infty$ limit [11]. This implies that the scaled gap probability

$$
E_{2}^{L \operatorname{hard}}(s ; a):=\lim _{N \rightarrow \infty} E_{2}^{L}\left(\frac{s}{4 N} ; a ; N\right)
$$

exists. Moreover, it was shown in [14] that for $a \in \mathbb{Z}_{\geqslant 0}, E_{2}^{L}(s ; a ; N)$ has a simple structure, allowing it, and its scaled limit, to be expressed as an $a \times a$ determinant. The determinant can alternatively be written as an $a$-dimensional integral, giving [10]

and thus relating to $(2.1)$.

$$
E_{2}^{L \text { hard }}(s ; a)=\mathrm{e}^{-s / 4}\left\langle\mathrm{e}^{\frac{1}{2} \sqrt{s} \operatorname{Tr}\left(U+U^{\dagger}\right)}\right\rangle_{U \in U(a)}
$$

The averages (2.2) and (2.3) have recently been shown to be equal to the cumulative distribution of the smallest eigenvalue in the scaled, infinite LOE and LSE respectively [17]. These matrix ensembles refer to the eigenvalue probability density function

$$
\frac{1}{C} \prod_{l=1}^{N} \lambda_{l}^{a} \mathrm{e}^{-c \lambda_{l} / 2} \prod_{1 \leqslant j<k \leqslant N}\left|\lambda_{k}-\lambda_{j}\right|^{\beta} \quad \lambda_{l}>0
$$

where $\beta=1, c=1$ for the LOE, and $\beta=4, c=2$ for the LSE. For $a=(n-N-1) / 2, n \geqslant N$, the LOE is realized by random matrices of the form $X^{T} X$, where $X$ is an $n \times N$ real standard Gaussian matrix, while for $a=2(n-N)+1$, matrices of the form $X^{\dagger} X$ with $X$ an $n \times N$ real quaternion Gaussian matrix (embedded as a complex matrix) realize the LSE (see, e.g., [13]). For general parameters $a, c, \beta$ in (2.9), analogous to (2.5), we define the gap probability

$E_{\beta}^{L}(s ; a, c ; N):=\frac{1}{C} \int_{s}^{\infty} \mathrm{d} \lambda_{1} \lambda_{1}^{a} \mathrm{e}^{-c \lambda_{1} / 2} \ldots \int_{s}^{\infty} \mathrm{d} \lambda_{N} \lambda_{N}^{a} \mathrm{e}^{-c \lambda_{N} / 2} \prod_{1 \leqslant j<k \leqslant N}\left|\lambda_{k}-\lambda_{j}\right|^{\beta}$.

As an aside we remark that a random matrix construction of the general $\beta$ Laguerre ensemble has recently been given [9]. For the scaled limits of the LOE and LSE cases, one defines

$$
\begin{aligned}
& E_{1}^{L \text { hard }}(s ; a):=\lim _{N \rightarrow \infty} E_{1}^{L}\left(\frac{s}{4 N} ; a, 1 ; N\right) \\
& E_{4}^{L \text { hard }}(s ; a):=\lim _{N \rightarrow \infty} E_{4}^{L}\left(\frac{s}{4 N} ; a, 2 ; N / 2\right)
\end{aligned}
$$


(in (2.12) it is assumed that $N$ is even; the use of $N / 2$ therein comes about naturally in studying the interrelationships between $E_{1}^{L}, E_{2}^{L}$ and $\left.E_{4}^{L}[16]\right)$. We know from [17] that

$$
\begin{aligned}
& E_{1}^{L \text { hard }}(s ; a)=\mathrm{e}^{-s / 8}\left\langle\mathrm{e}^{\frac{1}{2} \sqrt{s} \operatorname{Tr} S}\right\rangle_{S \in S p(a)} \\
& E_{4}^{L \text { hard }}(s ; 2 a)=\mathrm{e}^{-s / 8}\left\langle\mathrm{e}^{\frac{1}{2} \sqrt{s} \operatorname{Tr} O}\right\rangle_{O \in O(2 a)} .
\end{aligned}
$$

The derivation of (2.8) in [10] is different from the derivations of (2.13) and (2.14) in [17]. A unifying derivation can be given, based on the properties of zonal polynomials and corresponding hypergeometric functions, which we will now present.

The zonal polynomials are the special cases $\alpha=1 / 2,1$ and 2 of more general polynomials - the Jack polynomials - which depend on a continuous parameter $\alpha$. Let us then revise the definition of these polynomials. Let $\kappa:=\left(\kappa_{1}, \ldots, \kappa_{N}\right)$ denote a partition so that $\kappa_{i} \geqslant \kappa_{j}(i<j)$ and $\kappa_{i} \in \mathbb{Z}_{\geqslant 0}$. The modulus of a partition is defined by $|\kappa|:=\sum_{i=1}^{N} \kappa_{i}$. Let $m_{\kappa}$ denote the monomial symmetric function corresponding to the partition $\kappa$, and for partitions $|\kappa|=|\mu|$ define the dominance partial ordering by the statement that $\kappa>\mu$ if $\kappa \neq \mu$ and $\sum_{j=1}^{p} \kappa_{j} \geqslant \sum_{j=1}^{p} \mu_{j}$ for each $p=1, \ldots, N$. Introduce the Jack polynomials $P_{\kappa}^{(1 / \alpha)}\left(z_{1}, \ldots, z_{N}\right)=: P_{\kappa}^{(1 / \alpha)}(z)$ as the unique homogeneous polynomials of degree $|\kappa|$ with the structure

$$
P_{\kappa}^{(1 / \alpha)}(z)=m_{\kappa}+\sum_{\mu<\kappa} a_{\kappa \mu} m_{\mu}
$$

(the $a_{\kappa \mu}$ are some coefficients in $\mathbb{Q}(\alpha)$ ) and which satisfy the orthogonality

$$
\left\langle P_{\kappa}^{(1 / \alpha)}, P_{\rho}^{(1 / \alpha)}\right\rangle^{(\alpha)} \propto \delta_{\kappa, \rho}
$$

where

$$
\begin{gathered}
\langle f, g\rangle^{(\alpha)}:=\int_{-1 / 2}^{1 / 2} \mathrm{~d} x_{1} \cdots \int_{-1 / 2}^{1 / 2} \mathrm{~d} x_{N} \overline{f\left(z_{1}, \ldots, z_{N}\right)} g\left(z_{1}, \ldots, z_{N}\right) \prod_{1 \leqslant j<k \leqslant N}\left|z_{k}-z_{j}\right|^{2 \alpha} \\
z_{j}:=\mathrm{e}^{2 \pi \mathrm{i} \theta_{j}} .
\end{gathered}
$$

We remark that when $\alpha=1$ the Jack polynomials coincide with the Schur polynomials; also we should point out that there are other ways to define the Jack polynomials (see, e.g., [25]). Let

$$
d_{\kappa}^{\prime}=\prod_{(i, j) \in \kappa}(\alpha(a(i, j)+1)+l(i, j))
$$

where the notation $(i, j) \in \kappa$ refers to the diagram of $\kappa$, in which each part $\kappa_{i}$ becomes the nodes $(i, j), 1 \leqslant j \leqslant \kappa_{i}$, on a square lattice labelled as is conventional for a matrix. The quantity $a(i, j)$ is the so-called arm length (the number of nodes in row $i$ to the right of column $j$ ), while $l(i, j)$ is the leg length (number of nodes in column $j$ below row $i$ ). Define the renormalized Jack polynomial

$$
C_{\kappa}^{(\alpha)}(z):=\frac{\alpha^{|\kappa|}|\kappa| !}{d_{\kappa}^{\prime}} P_{\kappa}^{(\alpha)}(z)
$$

and introduce the generalized factorial function

$$
[u]_{\kappa}^{(\alpha)}=\prod_{j=1}^{N} \frac{\Gamma\left(u-(j-1) / \alpha+\kappa_{j}\right)}{\Gamma(u-(j-1) / \alpha)} .
$$


Then the generalized hypergeometric function ${ }_{p} F_{q}^{(\alpha)}$ based on the Jack polynomial (2.16) is specified by the series

$$
{ }_{p} F_{q}^{(\alpha)}\left(a_{1}, \ldots, a_{p} ; b_{1}, \ldots, b_{q} ; z\right):=\sum_{|\kappa|} \frac{1}{|\kappa| !} \frac{\left[a_{1}\right]_{\kappa}^{(\alpha)} \cdots\left[a_{p}\right]_{\kappa}^{(\alpha)}}{\left[b_{1}\right]_{\kappa}^{(\alpha)} \cdots\left[b_{q}\right]_{\kappa}^{(\alpha)}} C_{\kappa}^{(\alpha)}(z)
$$

(when $N=1$ this reduces to the classical definition of ${ }_{p} F_{q}$ ). For future reference, we draw attention to the confluence property

$$
\lim _{N \rightarrow \infty}{ }_{2} F_{1}^{(\alpha)}\left(N+a_{1}, N+a_{2} ; b ; z / N^{2}\right)={ }_{0} F_{1}^{(\alpha)}(b ; z)
$$

which is derived by recalling the homogeneity property $C_{\kappa}^{(\alpha)}\left(z / N^{2}\right)=N^{-2|\kappa|} C_{\kappa}^{(\alpha)}(z)$ and taking the limit term by term (the latter is justified since ${ }_{2} F_{1}^{(\alpha)}$ is analytic for $\left|z_{i}\right|<1, i=$ $1, \ldots, N[23])$.

In the special cases $\alpha=1 / 2,1$ and 2, the renormalized Jack polynomials $C_{\kappa}^{(\alpha)}(z)$ are zonal polynomials for Hermitian matrices with real, complex and real quaternion elements respectively (see, e.g., [25]). With $X$ an Hermitian matrix one defines $C_{\kappa}^{(\alpha)}(X)=$ $C_{\kappa}^{(\alpha)}\left(\lambda_{1}, \ldots, \lambda_{N}\right)$ where $\lambda_{1}, \ldots, \lambda_{N}$ are the eigenvalues of $X$ (the eigenvalues of a Hermitian matrix with real quaternion elements are doubly degenerate-in this case only the distinct eigenvalues are included). The zonal polynomials have a number of special properties not shared by the Jack polynomials in general. In particular, one has [18, 25, 29]

$$
\begin{aligned}
& \left\langle s_{\lambda}(A O)\right\rangle_{O \in O(n)}= \begin{cases}\frac{C_{\kappa}^{(2)}\left(A A^{T}\right)}{C_{\kappa}^{(2)}\left(1^{n}\right)} & 2 \kappa=\lambda \\
0 & \text { otherwise }\end{cases} \\
& \left\langle s_{\lambda}(A U) s_{\kappa}\left(U^{\dagger} A^{\dagger}\right)\right\rangle_{U \in U(n)}=\delta_{\lambda, \kappa} \frac{C_{\kappa}^{(1)}\left(A A^{\dagger}\right)}{C_{\kappa}^{(1)}\left(1^{n}\right)} \\
& \left\langle s_{\lambda}(A S)\right\rangle_{S \in S p(n)}= \begin{cases}\frac{C_{\kappa}^{(1 / 2)}\left(A A^{\dagger}\right)}{C_{\kappa}^{(1 / 2)}\left(1^{n}\right)} & \kappa^{2}=\lambda \\
0 & \text { otherwise }\end{cases}
\end{aligned}
$$

where in (2.19) $2 \kappa$ is the partition obtained by doubling each part of $\kappa$, while in $(2.21)$, $\kappa^{2}$ is the partition obtained by repeating each part of $\kappa$ twice. Also $C_{\kappa}^{(\alpha)}\left(1^{n}\right):=$ $\left.C_{\kappa}^{(\alpha)}\left(z_{1}, \ldots, z_{n}\right)\right|_{z_{1}=\cdots=z_{n}=1}$. As an aside we note that these zonal polynomial identities have recently been conjectured to carry over to the more general $q$-setting [31].

Of interest to us is a corollary of (2.19)-(2.21).

Corollary 1. We have

$$
\begin{aligned}
& \left\langle\mathrm{e}^{\operatorname{Tr}(A U)} \mathrm{e}^{\operatorname{Tr}\left(U^{\dagger} A^{\dagger}\right)}\right\rangle_{U \in U(n)}={ }_{0} F_{1}^{(1)}\left(n ; A A^{\dagger}\right) \\
& \left\langle\mathrm{e}^{\operatorname{Tr}(A S)}\right\rangle_{S \in S p(n)}={ }_{0} F_{1}^{(1 / 2)}\left(2 n ; A A^{\dagger}\right) \\
& \left\langle\mathrm{e}^{\operatorname{Tr}(A O)}\right\rangle_{O \in O(n)}={ }_{0} F_{1}^{(2)}\left(n / 2 ; A A^{T} / 4\right) .
\end{aligned}
$$

Proof. Now we know that for general $\alpha$ [34]

$$
C_{\kappa}^{(\alpha)}\left(1^{n}\right)=|\kappa| ! \frac{\alpha^{2|\kappa|}[n / \alpha]_{\kappa}^{(\alpha)}}{h_{\kappa} d_{\kappa}^{\prime}}
$$


where $d_{\kappa}^{\prime}$ is specified by (2.15) and

$$
h_{\kappa}:=\prod_{(i, j) \in \kappa}(\alpha a(i, j)+l(i, j)+1) .
$$

We also know that (see, e.g., [25])

$$
\sum_{\lambda} \frac{s_{\lambda}(X)}{\left.d_{\lambda}^{\prime}\right|_{\alpha=1}}=\exp \operatorname{Tr} X
$$

Consider the identities (2.19) and (2.21). We multiply both sides by $1 /\left.d_{\lambda}^{\prime}\right|_{\alpha=1}$ and use (2.26) on the left-hand sides. On the right-hand sides we use (2.25) and the easily verified identities

$$
\frac{\left.\left(h_{\kappa} d_{\kappa}^{\prime}\right)\right|_{\alpha=2}}{\left.d_{2 \kappa}^{\prime}\right|_{\alpha=1}}=1 \quad \frac{\left.2^{2|\kappa|}\left(h_{\kappa} d_{\kappa}^{\prime}\right)\right|_{\alpha=1 / 2}}{d_{\kappa^{2}}^{\prime}}=1
$$

together with the definition (2.17) to deduce (2.23) and (2.24). The identity (2.22) results by first multiplying both sides of $(2.20)$ by $1 /\left(\left.d_{\lambda}^{\prime}\right|_{\alpha=1}\right)^{2}$, making use of (2.25) (note that for $\left.\alpha=1, d_{\kappa}^{\prime}=h_{\kappa}\right)$, then using (2.26) and (2.17).

We remark that the first and third identities of corollary 1 are due to James [18], while the second, which is implicit in the work of Rains [29], seems not to have appeared in the print before.

The probability $E_{\beta}^{L}(s ; a, c ; N)$ as specified by (2.10), for general $\beta>0$, general $N$, scale chosen with $c=\beta$ and $a \in \mathbb{Z}_{\geqslant 0}$ has been given in [10] in terms of the generalized hypergeometric function ${ }_{1} F_{1}^{(\beta / 2)}$. In the scaled $N \rightarrow \infty$ limit this same probability was given in terms of the generalized hypergeometric function ${ }_{0} F_{1}^{(\beta / 2)}$. Thus, one has

$$
E_{\beta}^{L \text { hard }}(s ; a, \beta):=\lim _{N \rightarrow \infty} E_{\beta}^{L}\left(\frac{s}{4 N} ; a, \beta ; N\right)=\left.\mathrm{e}_{0}^{-\beta s / 8} F_{1}^{(\beta / 2)}\left(\frac{2 a}{\beta} ; x_{1}, \ldots, x_{a}\right)\right|_{x_{1}=\cdots=x_{a}=s / 4} .
$$

For $\beta=1$ and $\beta=2$ the definition of $E_{\beta}^{L \text { hard }}(s ; a, \beta)$ coincides with the definition of $E_{1}^{L \text { hard }}(s ; a)$ given by $(2.11)$ and $E_{2}^{L \text { hard }}(s ; a)$ given by $(2.7)$ respectively, so we have

$$
\begin{aligned}
& E_{1}^{L \text { hard }}(s ; a)=\left.\mathrm{e}^{-s / 8}{ }_{0} F_{1}^{(1 / 2)}\left(2 a ; x_{1}, \ldots, x_{a}\right)\right|_{x_{1}=\cdots=x_{a}=s / 4} \\
& E_{2}^{L \text { hard }}(s ; a)=\left.\mathrm{e}^{-s / 4}{ }_{0} F_{1}^{(1)}\left(a ; x_{1}, \ldots, x_{a}\right)\right|_{x_{1}=\cdots=x_{a}=s / 4} .
\end{aligned}
$$

However, in the case $\beta=4$ the choice $c=\beta$ used to derive (2.28) does not agree with the convention used to specify $E_{4}^{L}$ hard $(s ; a)$ in (2.12), which also had the peculiarity of first having $N$ replaced by $N / 2$ before the $N \rightarrow \infty$ limit is taken. As a result, we have

$$
E_{4}^{L \text { hard }}(s ; a)=\left.E_{\beta}^{L \text { hard }}(s / 4 ; a, c)\right|_{\beta=c=4}
$$

and consequently

$$
E_{4}^{L \text { hard }}(s ; a)=\left.\mathrm{e}^{-s / 8} F_{1}^{(2)}\left(a / 2 ; x_{1}, \ldots, x_{a}\right)\right|_{x_{1}=\cdots=x_{a}=s / 16} .
$$

The identities (2.8), (2.13) and (2.14) can now be reclaimed. Thus, in corollary 1 we choose $A=(\sqrt{t} / 2) I_{n}, I_{n}$ denoting the $n \times n$ identity matrix, and substitute the resulting forms in (2.30), (2.29) and (2.32) respectively.

With this side issue resolved, let us now explicitly state the implication of the identities (2.8), (2.13) and (2.14) in relation to the probabilities $\operatorname{Pr}\left(L^{\square}(t) \leqslant l\right), \operatorname{Pr}\left(L^{\square}(t) \leqslant l\right)$ and $\operatorname{Pr}\left(L^{\square}(t) \leqslant l\right)$. 
Proposition 1. We have

$$
\begin{aligned}
& \operatorname{Pr}\left(L^{\square}(t / 4) \leqslant l\right)=E_{2}^{L \text { hard }}(t ; l) \\
& \operatorname{Pr}\left(L^{\square}(t / 4) \leqslant 2 l\right)=E_{1}^{L \text { hard }}(t ; l) \\
& \operatorname{Pr}\left(L^{\square}(t / 4) \leqslant 2 l\right)=E_{4}^{L \text { hard }}(t ; 2 l) .
\end{aligned}
$$

Proof. Substitute (2.8), (2.13) and (2.14) in the identities (2.1)-(2.3) respectively, and then substitute the new form of (2.1)-(2.3) in (1.3), (1.5) and (1.6) respectively.

\section{The gap probabilities as a sum over $k$-point correlations}

We seek formulae for the hard edge gap probability in proposition 1 which enable the scaled $t, l \rightarrow \infty$ limit to be analysed. For this purpose we make use of the well-known (and simple to derive) fact that for a general eigenvalue probability density function the probability $E^{(N)}(I)$ of having no eigenvalues in an interval $I$ is given as a sum over the corresponding $k$-point correlations,

$$
E^{(N)}(I)=1+\sum_{k=1}^{N} \frac{(-1)^{k}}{k !} \int_{I} \mathrm{~d} x_{1} \cdots \int_{I} \mathrm{~d} x_{k} \rho_{k}^{(N)}\left(x_{1}, \ldots, x_{k}\right) .
$$

Let $A_{N}(x)=\alpha_{N}+a_{N} x$ define a linear scale such that for $x_{1}, \ldots, x_{k}$ fixed

$$
\lim _{N \rightarrow \infty} a_{N}^{k} \rho_{k}^{(N)}\left(A_{N}\left(x_{1}\right), \ldots, A_{N}\left(x_{k}\right)\right)=\rho_{k}\left(x_{1}, \ldots, x_{k}\right)
$$

where $\rho_{k}$ denotes the limiting distribution. We would like to write $\lim _{N \rightarrow \infty} E^{(N)}\left(A_{N}(I)\right)$ as the right-hand side of (3.1) with $\rho_{k}^{(N)}$ replaced by $\rho_{k}$. Sufficient conditions for this to hold are given by the following specialization (and minor rewrite) of a recent lemma due to Soshnikov [33, lemma 2].

Proposition 2. Consider a sequence of point processes labelled by N. Suppose that after the linear scaling $x_{j} \mapsto A_{N}\left(x_{j}\right)$ of each of the coordinates, the sequence approaches a limit point process with correlations $\left\{\rho_{k}\right\}_{k=1,2, \ldots}$ such that

$$
\sum_{k=1}^{\infty}\left(\frac{1}{k !} \int_{I} \mathrm{~d} x_{1} \cdots \int_{I} \mathrm{~d} x_{k} \rho_{k}\left(x_{1}, \ldots, x_{k}\right)\right)^{-1 / k}
$$

diverges, and suppose furthermore that

$$
\begin{gathered}
\lim _{N \rightarrow \infty} a_{N}^{k} \int_{A_{N}(I)} \mathrm{d} x_{1} \cdots \int_{A_{N}(I)} \mathrm{d} x_{k} \rho_{k}^{(N)}\left(A_{N}\left(x_{1}\right), \ldots, A_{N}\left(x_{k}\right)\right) \\
=\int_{I} \mathrm{~d} x_{1} \cdots \int_{I} \mathrm{~d} x_{k} \rho_{k}\left(x_{1}, \ldots, x_{k}\right) .
\end{gathered}
$$

Then

$E(I):=\lim _{N \rightarrow \infty} E^{(N)}\left(A_{N}(I)\right)=1+\sum_{k=1}^{\infty} \frac{(-1)^{k}}{k !} \int_{I} \mathrm{~d} x_{1} \cdots \int_{I} \mathrm{~d} x_{k} \rho_{k}\left(x_{1}, \ldots, x_{k}\right)$.

The significance of the condition that (3.3) diverges is that it implies [24, 32] the limit process to then have the property that for any $I \subset \mathbb{R}$ the distribution of the number of particles in $I$ is uniquely determined by the correlation functions of the process. More explicitly, the divergence of (3.3) implies that the moment problem for the number of particles in $I$ is definite, 
and thus the convergence of moments (which are integrals of correlation functions) implies the convergence of distributions. Observe that a sufficient condition for (3.3) to diverge is that

$$
\int_{I} \mathrm{~d} x_{1} \cdots \int_{I} \mathrm{~d} x_{k} \rho_{k}\left(x_{1}, \ldots, x_{k}\right)=o(k !)
$$

which itself is required for the series in (3.5) to be convergent.

Let us now compute the explicit form of (3.5) in the case of the LUE hard edge gap probability. The form of $\rho_{k}^{(N)}$ in this case has a structure common to all probability density functions of the form

$$
\frac{1}{C} \prod_{l=1}^{N} w_{2}\left(\lambda_{l}\right) \prod_{1 \leqslant j<k \leqslant N}\left(\lambda_{k}-\lambda_{j}\right)^{2}
$$

Thus, with $w_{2}(x)$ in (3.7) non-negative but otherwise general the corresponding $k$-point correlations are given by

$$
\rho_{k}^{(N)}\left(x_{1}, \ldots, x_{k}\right)=\operatorname{det}\left[K\left(x_{j}, x_{l}\right)\right]_{j, l=1, \ldots, k}
$$

where

$$
K(x, y):=\frac{\left(w_{2}(x) w_{2}(y)\right)^{1 / 2}}{\left(p_{N-1}, p_{N-1}\right)_{2}} \frac{p_{N}(x) p_{N-1}(y)-p_{N}(y) p_{N-1}(x)}{x-y} .
$$

In (3.9) $\left\{p_{j}(x)\right\}_{j=0,1, \ldots}$ is the set of monic polynomials orthogonal with respect to $w_{2}(x)$, and $\left(p_{n}, p_{n}\right)_{2}$ is the corresponding normalization. The LUE is the special case $w_{2}(x)=$ $x^{a} \mathrm{e}^{-x}(x>0)$ of (3.7). Denote (3.9) in this case by $K^{L}$. Then we know [11] that for fixed $x, y>0$

$$
\begin{aligned}
& \lim _{N \rightarrow \infty} \frac{1}{4 N} K^{L}\left(\frac{x}{4 N}, \frac{y}{4 N}\right)=K_{2}^{\text {Bessel }}(x, y) \\
& K^{\text {Bessel }}(x, y):=\frac{J_{a}(\sqrt{x}) \sqrt{y} J_{a}^{\prime}(\sqrt{y})-\sqrt{x} J_{a}^{\prime}(\sqrt{x}) J_{a}(\sqrt{y})}{2(x-y)} .
\end{aligned}
$$

Furthermore, it has been proved [6] that the convergence in (3.10) is uniform for $x, y$ in compact sets on $[0, \infty)$. Because $I=[0, s]$ is compact, it follows immediately that (3.4) holds with $a_{N}=1 / 4 N$. The fact that $\rho_{k}$ is given by the determinant of a $k \times k$ symmetric non-negative matrix, the entries of which are independent of $k$, implies the bound [21]

$$
\rho_{k}\left(x_{1}, \ldots, x_{k}\right) \leqslant \rho_{1}\left(x_{1}\right) \cdots \rho_{1}\left(x_{k}\right) .
$$

This in turn implies

$$
\int_{I} \mathrm{~d} x_{1} \cdots \int_{I} \mathrm{~d} x_{k} \rho_{k}\left(x_{1}, \ldots, x_{k}\right)=O\left(c^{k}\right)
$$

for some $c>0$, so (3.6) holds. Consequently, from proposition 2, the scaled gap probability (2.7) can be written in the following well-known form [36].

Proposition 3. Let $K_{2}^{\text {Bessel }}$ be given by (3.11). We have

$E_{2}^{L \text { hard }}(s ; a)=1+\sum_{k=1}^{\infty} \frac{(-1)^{k}}{k !} \int_{0}^{s} \mathrm{~d} x_{1} \cdots \int_{0}^{s} \mathrm{~d} x_{k} \operatorname{det}\left[K_{2}^{\text {Bessel }}\left(x_{j}, x_{l}\right)\right]_{j, l=1, \ldots, k}$.

In the cases $\beta=1$ and $\beta=4$ the limiting correlations are quaternion determinants, or equivalently Pfaffians $[8,15]$. This leads to a more complicated analysis than that required for the case $\beta=2$. However, at the expense of a minor digression, the computation of $E_{1}^{L \text { hard }}$ as 
a sum over correlations can also be posed as a problem involving (scalar) determinants rather than Pfaffians. In addition, by following this route we will reclaim the known fact [16] that $E_{4}^{L \text { hard }}(s ; a)$ is simply related to $E_{2}^{L \text { hard }}\left(s ; a_{2}\right)$ and $E_{1}^{L \text { hard }}\left(s ; a_{1}\right)$ for particular $a_{1}, a_{2}$, so no independent analysis of $E_{4}^{L \text { hard }}(s ; a)$ is required.

We begin our digression by revising that the general $\beta$ Laguerre ensemble as specified by (2.9) can be viewed as a limiting case of the Jacobi ensemble, the latter specified by the eigenvalue probability density function

$$
\frac{1}{C} \prod_{l=1}^{N} \lambda_{l}^{a}\left(1-\lambda_{l}\right)^{b} \prod_{1 \leqslant j<k \leqslant N}\left|\lambda_{k}-\lambda_{j}\right|^{\beta} \quad 0<\lambda_{l}<1 .
$$

For $\beta=1,2$ and 4 , and $a=\beta\left(n_{1}-m+1\right) / 2-1, b=\beta\left(n_{2}-m+1\right) / 2-1$, this is realized by matrices of the form $A(A+B)^{-1}$, where $A=a^{\dagger} a, B=b^{\dagger} b$, with $a, b$ real $(\beta=1)$, complex $(\beta=2)$ and real quaternion $(\beta=4)$ Gaussian random matrices of dimensions $n_{1} \times m, n_{2} \times m$ $[13,26]$. To obtain (2.9) from (3.14), make the replacement $\lambda_{l} \mapsto c \lambda_{l} / 2 b$ in (3.14) and take the limit $b \rightarrow \infty$. In the vicinity of $\lambda=0$ both (2.9) and (3.14) have the same functional form, and so it is to be anticipated that after appropriate scaling the local statistical properties will also be the same. In the case of the hard edge gap probability, this can readily be demonstrated, as we will now show.

Let $E_{\beta}^{J}(s ; a, b ; N)$ denote the probability that there are no eigenvalues in the interval $(0, s)$ of the Jacobi ensemble (3.14). Then, by definition

$$
\begin{aligned}
E_{\beta}^{J}(s ; a, b ; N) & =\frac{1}{C} \int_{s}^{1} \mathrm{~d} \lambda_{1} \lambda_{1}^{a}\left(1-\lambda_{1}\right)^{b} \cdots \int_{s}^{1} \mathrm{~d} \lambda_{N} \lambda_{N}^{a}\left(1-\lambda_{N}\right)^{b} \prod_{1 \leqslant j<k \leqslant N}\left|\lambda_{k}-\lambda_{j}\right|^{\beta} \\
= & \frac{1}{C}(1-s)^{(1+a+b) N+\beta N(N-1) / 2} \int_{0}^{1} \mathrm{~d} \lambda_{1}\left(\lambda_{1}+s /(1-s)\right)^{a}\left(1-\lambda_{1}\right)^{b} \\
& \cdots \int_{0}^{1} \mathrm{~d} \lambda_{N}\left(\lambda_{N}+s /(1-s)\right)^{a}\left(1-\lambda_{N}\right)^{b} \prod_{1 \leqslant j<k \leqslant N}\left|\lambda_{k}-\lambda_{j}\right|^{\beta} .
\end{aligned}
$$

Note that for positive integer values of $a$ the multidimensional integral in this last expression is a polynomial in $s /(1-s)$. From the work of Kaneko [23] we know that this multidimensional integral can be written as an $a$-dimensional generalized hypergeometric function ${ }_{2} F_{1}^{(\beta / 2)}$, thus giving

$$
\begin{aligned}
E_{\beta}^{J}(s ; a, b ; N) & =(1-s)^{(1+a+b) N+\beta N(N-1) / 2} \\
& \times\left.{ }_{2} F_{1}^{(\beta / 2)}\left(-N ; \frac{2}{\beta}(a+b+1)+N-1, \frac{2}{\beta} a ; s_{1}, \ldots, s_{a}\right)\right|_{s_{1}=\cdots=s_{a}=-s /(1-s)}
\end{aligned}
$$

(the normalization is fixed by requiring that both sides equal unity for $s=0$ ). The scaled $N \rightarrow \infty$ limit can now be read off using (2.18).

Proposition 4. For $a \in \mathbb{Z}_{\geqslant 0}$,

$$
E_{\beta}^{J \text { hard }}(s ; a):=\lim _{N \rightarrow \infty} E_{\beta}^{J \text { hard }}\left(\frac{s}{4 N^{2}} ; a, b ; N\right)=\left.\mathrm{e}^{-\beta s / 8}{ }_{0} F_{1}^{(\beta / 2)}\left(\frac{2 a}{\beta} ; s_{1}, \ldots, s_{a}\right)\right|_{s_{1}=\cdots=s_{a}=s / 4} .
$$

Comparing with (2.28) we see that

$$
E_{\beta}^{J \text { hard }}(s ; a)=E_{\beta}^{L \text { hard }}(s ; a, \beta)
$$


as anticipated. Thus, we can drop the superscripts $J$ and $L$ and simply write $E_{\beta}^{\text {hard }}(s ; a)$, where it is to be understood that in the case $\beta=4$ we refer to the scaling (2.12).

There is an advantage in working with the Jacobi ensemble rather than the Laguerre ensemble. This comes about because of special features of the case $b=0$ of the former. One such special feature is the formula [16]

$E_{4}^{J}(s / 2 ; a+1,0 ; N / 2)=\frac{1}{2}\left(E_{1}^{J}(s ;(a-1) / 2,0 ; N)+\frac{E_{2}^{J}(s ; a, 0 ; N)}{E_{1}^{J}(s ;(a-1) / 2,0 ; N)}\right)$.

Taking the scaled limit on both sides shows [16]

$$
E_{4}^{\text {hard }}(s ; a+1)=\frac{1}{2}\left(E_{1}^{\text {hard }}(s ;(a-1) / 2)+\frac{E_{2}^{\text {hard }}(s ; a)}{E_{1}^{\text {hard }}(s ;(a-1) / 2)}\right)
$$

thus reducing the study of the $\beta=4$ case down to that of the $\beta=1$ and 2 cases. We will see that the analysis of $E_{1}^{\text {hard }}$ is also made easier by considering the Jacobi ensemble with $b=0$.

The simplified analysis of $E_{1}^{\text {hard }}$ from this perspective comes about because the ensemble $\left.\left.\mathrm{JOE}\right|_{b=0} \cup \mathrm{JOE}\right|_{b=0}=: J^{2}$, formed out of two independent copies of the JOE with $b=0$, has a simple determinant form for the $k$-point distribution of the odd labelled coordinates (with the eigenvalues ordered $0<x_{1}<x_{2}<\cdots<x_{2 N}<1$ ) [16],

$$
\begin{aligned}
& \left.\rho_{(k)}^{(N) \text { odd }}\left(x_{1}, \ldots, x_{k}\right)\right|_{a \mapsto(a-1) / 2}=\operatorname{det}\left[K^{J^{2}}\left(x_{j}, x_{l}\right)\right]_{j, l=1, \ldots, k} \\
& K^{J^{2}}(x, y):=-\frac{\partial}{\partial x}(1-x)^{1 / 2} \int_{0}^{y}(1-u)^{-1 / 2} K^{J}(x, u) \mathrm{d} u
\end{aligned}
$$

where $K^{J}$ is the function (3.9) in the Jacobi case with $b=0$ and the $a$ parameter left unchanged.

To make use of (3.21) we follow [12] and first note

$$
\left(E_{1}^{J}(s ; a, 0, N)\right)^{2}=E^{J^{2}}(s ; a, 0, N)=E^{\operatorname{odd}\left(J^{2}\right)}(s ; a, 0, N)
$$

where the first equality follows from the definition of the ensemble $J^{2}$, while the second equality follows from the fact that the eigenvalues have been labelled so that the first is closest to the edge at $x=0$. Hence, from (3.1) and (3.21)

$$
\left(E_{1}^{J}(s ;(a-1) / 2,0, N)\right)^{2}=1+\sum_{k=1}^{N} \frac{(-1)^{k}}{k !} \int_{0}^{s} \mathrm{~d} x_{1} \cdots \int_{0}^{s} \mathrm{~d} x_{k} \operatorname{det}\left[K^{J^{2}}\left(x_{j}, x_{l}\right)\right]_{j, l=1, \ldots, k} .
$$

To proceed further we require the scaled limit of $K^{J^{2}}(x, y)$.

Proposition 5. We have

$$
\begin{aligned}
\lim _{N \rightarrow \infty} \frac{1}{4 N} K^{J^{2}} & \left(\frac{x}{4 N}, \frac{y}{4 N}\right) \\
& =\sqrt{\frac{y}{x}} K_{2}^{\text {Bessel }}(x, y)+\frac{J_{a}(\sqrt{x})}{2 \sqrt{x}}\left(1-\int_{0}^{\sqrt{y}} J_{a}(t) \mathrm{d} t\right)=: K_{1}^{\text {Bessel }}(x, y)
\end{aligned}
$$

where the convergence is uniform for $x, y \in(0, s)$.

Proof. Using standard uniform estimates for the Jacobi polynomials, it has been shown in [16] that the left-hand side of (3.25) converges uniformly for $x, y \in(0, s)$ to

$$
\frac{\partial}{\partial x}\left(x^{1 / 2} \int_{y}^{\infty} v^{-1 / 2} K_{2}^{\text {Bessel }}(x, v) \mathrm{d} v\right) .
$$


In the same reference, an identity equivalent to the equality between this expression and $K_{1}^{\text {Bessel }}$ has been given.

It follows from proposition 5 and (3.21) that in addition to the pointwise convergence of the correlations, the stronger convergence property (3.4) also holds. Furthermore, noting that $K_{1}^{\text {Bessel }}$ is bounded for $x, y \in[0, s]$ (by $M$ say), and Hadamards lemma on bounds for determinants [38] implies that

$$
\rho_{k}\left(x_{1}, \ldots, x_{k}\right) \leqslant k^{k / 2} M^{k}
$$

(note that the bound (3.12) no longer necessarily holds because $K_{1}^{\text {Bessel }}$ is not symmetric). The inequality (3.26), although a gross overestimate of the physically plausible $\rho_{k} \leqslant C^{k}$, is still sufficient to establish (3.6), so we have that both criteria sufficient for the validity of (3.5) hold. Hence, the scaled gap probability can be expanded in the following form, known but not rigorously justified in [12].

Proposition 6. We have

$\left(E_{1}^{\mathrm{hard}}(s ;(a-1) / 2)\right)^{2}=1+\sum_{k=1}^{\infty} \frac{(-1)^{k}}{k !} \int_{0}^{s} \mathrm{~d} x_{1} \cdots \int_{0}^{s} \mathrm{~d} x_{k} \operatorname{det}\left[K_{1}^{\text {Bessel }}\left(x_{j}, x_{l}\right)\right]_{j, l=1, \ldots, k}$

where $K_{1}^{\text {Bessel }}(x, y)$ is given by (3.25).

Our final preliminary task is to present formulae analogous to (3.13), (3.20) and (3.27) for the scaled probabilities $F_{2}(s), F_{1}(s)$ and $F_{4}(s)$ occurring on the right-hand side of (1.1), (1.5) and (1.7) respectively. By definition $F_{1}(s), F_{2}(s)$ and $F_{4}(s)$ are the scaled probabilities of no eigenvalues at the edge of the spectrum of the Gaussian $\beta$-ensemble, with $\beta=1,2$ and 4 respectively, the latter being specified by the eigenvalue probability density function

$$
\frac{1}{C} \prod_{l=1}^{N} \mathrm{e}^{-c x_{l}^{2} / 2} \prod_{1 \leqslant j<k \leqslant N}\left|x_{j}-x_{k}\right|^{\beta} .
$$

Explicitly define

$$
E_{\beta}^{G}(s ; \beta, c ; N)=\frac{1}{C} \int_{-\infty}^{s} \mathrm{~d} x_{1} \cdots \int_{-\infty}^{s} \mathrm{~d} x_{N} \prod_{l=1}^{N} \mathrm{e}^{-c x_{l}^{2} / 2} \prod_{1 \leqslant j<k \leqslant N}\left|x_{k}-x_{j}\right|^{\beta} .
$$

Then

$$
\begin{aligned}
& F_{1}(s):=\lim _{N \rightarrow \infty} E_{1}^{G}\left(\sqrt{2 N}+s / \sqrt{2} N^{1 / 6} ; 1,1 ; N\right) \\
& F_{2}(s):=\lim _{N \rightarrow \infty} E_{2}^{G}\left(\sqrt{2 N}+s / \sqrt{2} N^{1 / 6} ; 2,2 ; N\right) \\
& F_{4}(s):=\lim _{N \rightarrow \infty} E_{2}^{G}\left(\sqrt{2 N}+s / \sqrt{2} N^{1 / 6} ; 4,2 ; N / 2\right) .
\end{aligned}
$$

In general, choosing $(c, N) \mapsto(1, N),(2, N),(2, N / 2)$ for $\beta=1,2$ and 4 in (3.28) and then scaling the coordinates by

$$
x_{l} \mapsto \sqrt{2 N}+\frac{x_{l}}{\sqrt{2} N^{1 / 6}}
$$

gives the so-called 'soft edge' process with parameter $\beta$. The limiting $k$-point correlation functions have been explicitly computed as a $k \times k$ determinant in the case $\beta=2$ [11], 
and a $k \times k$ quaternion determinant (or equivalently Pfaffian) in the cases $\beta=1$ and $\beta=4$ [15]. Moreover, the uniform asymptotic expansion of the Hermite polynomials [28]

$$
\mathrm{e}^{-x^{2} / 2} H_{N}(x)=\pi^{-3 / 4} 2^{N / 2+1 / 4}(N !)^{-1 / 12}\left\{\pi \operatorname{Ai}(t)+O\left(\mathrm{e}^{-t}\right) O\left(N^{-2 / 3}\right)\right\}
$$

where $x=(2 N)^{1 / 2}+t / 2^{1 / 2} N^{1 / 6}, t \in\left[t_{0}, \infty\right)$, shows that the correlation functions converge not only pointwise, but also in the sense of (3.4). In particular, in the case $\beta=2$ this convergence, together with the bound (3.12), implies the well-known formula [35]

$$
F_{2}(s)=1+\sum_{k=1}^{\infty} \frac{(-1)^{k}}{k !} \int_{s}^{\infty} \mathrm{d} x_{1} \cdots \int_{s}^{\infty} \mathrm{d} x_{k} \operatorname{det}\left[K_{2}^{\text {Airy }}\left(x_{j}, x_{l}\right)\right]_{j, l=1, \ldots, k}
$$

where

$$
K_{2}^{\text {Airy }}(x, y)=\frac{\operatorname{Ai}(x) \operatorname{Ai}^{\prime}(y)-\operatorname{Ai}(y) \operatorname{Ai}^{\prime}(x)}{x-y} .
$$

In the cases $\beta=1$ and $\beta=4$ this line of reasoning gives an expansion for $F_{\beta}(s)$ of the form (3.32), but involving a quaternion determinant in place of the scalar determinant in the case $\beta=2$. However, analogous to (3.27), in the case $\beta=1$ an alternative expansion involving a scalar determinant can be derived [12],

$$
\begin{aligned}
& \left(F_{1}(s)\right)^{2}=1+\sum_{k=1}^{\infty} \frac{(-1)^{k}}{k !} \int_{s}^{\infty} \mathrm{d} x_{1} \cdots \int_{s}^{\infty} \mathrm{d} x_{k} \operatorname{det}\left[K_{1}^{\text {Airy }}\left(x_{j}, x_{l}\right)\right]_{j, l=1, \ldots, k} \\
& K_{1}^{\text {Airy }}(x, y)=K_{2}^{\text {Airy }}(x, y)+\operatorname{Ai}(x)\left(1-\int_{y}^{\infty} \operatorname{Ai}(v) \mathrm{d} v\right) .
\end{aligned}
$$

The workings in [12] leading to (3.34) are formal rather than rigorous. Nonetheless, in the spirit of the chain of argument leading to (3.27), a rigorous derivation of (3.34) can be given. Before doing this we remark that the right-hand side of (3.35) is formally the expansion of the Fredholm determinant of $1+K_{1}^{\text {Airy }}$, where $K_{1}^{\text {Airy }}$ is the integral operator on $(s, \infty)$ with kernel $K_{1}^{\text {Airy }}(x, y)$. However, as $K_{1}^{\text {Airy }}(x, y)$ does not go to zero for $x$ fixed and $y \rightarrow \infty, K_{1}^{\text {Airy }}$ does not define a bounded operator, so in fact det $\left(1+K_{1}^{\text {Airy }}\right)$ is not a Fredholm determinant. But still the series (3.34) is convergent, as can be seen by replacing $K_{1}^{\text {Airy }}\left(x_{j}, x_{l}\right)$ in the determinant by $\mathrm{e}^{x_{j}-x_{l}} K_{1}^{\text {Airy }}\left(x_{j}, x_{l}\right)$ (this leaves the value of the determinant unchanged) and noting the decay of $\mathrm{e}^{x-y} K_{1}^{\text {Airy }}(x, y)$ as $x$ and/or $y$ approach $\infty$.

Returning now to the task at hand, we recall that in the rigorous derivation of (3.27) presented above, instead of working with the Laguerre ensemble, a particular Jacobi ensemble was analysed. This was permitted because it could be established that the limiting hard edge probability is the same in both the Laguerre and Jacobi ensembles. Likewise we undertake the task of a rigorous derivation of (3.34) by analysing not the finite $N$ GOE, but rather the finite $N$ LOE with $a=0$. The asymptotic analysis of [16] shows that with the linear change of scale in this latter ensemble,

$$
x_{l} \mapsto 4 N+2(2 N)^{1 / 3} x_{l}
$$

the correlation functions converge to the limiting GOE soft edge correlations in the sense of (3.4). Thus from proposition 2 we can regard $F_{1}(s)$ as the scaled limit of $E_{1}^{L}((s, \infty) ; 0, N)$ where the latter denotes the probability that there are no eigenvalues in the interval $(s, \infty)$ of the finite $N$ LOE with $a=0$. The use of this perspective, analogous to the use of working with the $b=0$ Jacobi ensemble rather than the Laguerre ensemble at the hard edge, is that the ensemble $\left.\left.\mathrm{LOE}\right|_{a=0} \cup \mathrm{LOE}\right|_{a=0}=: L^{2}$, formed out of two independent copies of the LOE 
with $a=0$, has a simple determinant form for the $k$-point correlation of the even labelled coordinates (with eigenvalues ordered $0<x_{1}<x_{2}<\cdots<x_{2 N}<\infty$ ) [16],

$$
\begin{aligned}
& \rho_{(k)}^{(N) \operatorname{even}}\left(x_{1}, \ldots, x_{k}\right)=\operatorname{det}\left[K^{L^{2}}\left(x_{j}, x_{l}\right)\right]_{j, l=1, \ldots, k} \\
& K^{L^{2}}(x, y):=-\frac{\partial}{\partial x} \int_{0}^{y} K^{L}(x, u) \mathrm{d} u
\end{aligned}
$$

where $K^{L}$ is the function (3.9) in the Laguerre case with $a=0$. To make use of (3.37), we note

$$
\left(E_{1}^{L}((s, \infty) ; 0, N)\right)^{2}=E^{L^{2}}((s, \infty) ; 0, N)=E^{\operatorname{even}\left(L^{2}\right)}((s, \infty) ; 0, N)
$$

(cf (3.23)). Now we seek the scaled limit of $K^{L^{2}}$ and thus the scaled limit of $\rho_{k}^{\text {even }}$.

Proposition 7. Let $K_{1}^{\text {Airy }}(x, y)$ be given by (3.35). We have

$\lim _{N \rightarrow \infty} 2(2 N)^{1 / 3} K^{L^{2}}\left(4 N+2(2 N)^{1 / 3} x, 4 N+2(2 N)^{1 / 3} y\right)=K_{1}^{\text {Airy }}(x, y)$

where the convergence is uniform on $x, y \in(s, \infty)$ with remainder terms which decay exponentially fast in $x$.

Proof. This result is essentially contained in [16]. Thus, using the uniform asymptotic expansion

$$
\mathrm{e}^{-x / 2} x^{a / 2} L_{n}^{a}(x)=n^{a / 2}\left(\frac{(-1)^{n}}{2^{a}(2 n)^{1 / 3}} \operatorname{Ai}(t)+o\left(n^{-1 / 3}\right) O\left(\mathrm{e}^{-t}\right)\right)
$$

where $x=4 n+2+2(2 n)^{1 / 3} t, t \in\left[t_{0}, \infty\right)(\mathrm{cf}(3.31))$, in [16] uniform convergence to

$$
-\frac{\partial}{\partial x} \int_{-\infty}^{y} K_{2}^{\text {Airy }}(x, t) \mathrm{d} t
$$

is established, as is the equality between (3.41) and $K_{1}^{\text {Airy }}$. The uniform exponentially decaying bound on the error in (3.40) can be used to deduce that the remainder terms in the convergence of (3.39) decay exponentially fast in $x$. The structure of the required working is the same as in the proof of proposition 8 , so the details will not be presented.

The results of proposition 7 allow (3.34) to be established. Thus, in light of the relation (3.38), and the evaluation of the scaled limit of $\rho_{k}^{\text {even }}$ given by (3.37) and (3.39) we know from proposition 2 that (3.34) will be valid if the properties (3.5) and (3.6) can be verified. Now the structure of the convergence of $K^{L^{2}}$ in the scaled limit of (3.39) substituted in (3.37) shows immediately that the stronger convergence (3.5) holds true. Furthermore, recalling that $\operatorname{Ai}(X)=O\left(\mathrm{e}^{-2 x^{3 / 2} / 3}\right)$ as $x \rightarrow \infty$ we see that $\mathrm{e}^{x} K_{1}^{\text {Airy }}(x, y)$ is bounded for $x, y \in\left[s_{0}, \infty\right)$ (by $M$ say) and thus, making use also of Hadamards lemma, we have

$$
\rho_{k}\left(x_{1}, \ldots, x_{k}\right) \leqslant \mathrm{e}^{-\left(x_{1}+\cdots+x_{k}\right)} k^{k / 2} M^{k} .
$$

This inequality establishes (3.6), thus concluding the work to justify (3.34).

It remains to consider the soft edge gap probability for $\beta=4$. For this, we have the interrelationship analogous to (3.19) [16],

$$
F_{4}(s)=\frac{1}{2}\left(F_{1}(s)+\frac{F_{2}(s)}{F_{1}(s)}\right)
$$

which was derived as the limiting form of an exact interrelationship between the corresponding finite $N$ gap probabilities in the Gaussian ensembles. 


\section{The hard-to-soft edge transition}

Our objective in this section is to prove the following limit theorem.

Theorem 1. For $\beta=1,2$ let $E_{\beta}^{\text {hard }}(s ; a)$ denote the hard edge gap probability defined by (2.11) and (2.7), and let $F_{\beta}(s)$ denote the soft edge gap probability defined by (3.29). We have

$$
\lim _{a \rightarrow \infty} E_{\beta}^{\mathrm{hard}}\left(a^{2}-2 a(a / 2)^{1 / 3} s+O(a) ; c a / 2\right)=F_{\beta}(s),
$$

where $c=1$ for $\beta=1$ and $c=2$ for $\beta=2$.

Before discussing the proof of this theorem, let us first note an immediate corollary.

Corollary 2. Let $E_{4}^{\text {hard }}(s ; a)$ denote the hard edge gap probability defined by $(2.12)$, and $F_{4}(s)$ denote the soft edge gap probability defined in (3.29). We have that the limit relation (4.1) with $c=2$ holds for $\beta=4$.

Proof. We substitute $s \mapsto a^{2}-2 a(a / 2)^{1 / 3} s+O(a)$ in (3.19), and take the limit $a \rightarrow \infty$ on the right-hand side by using (4.1). The resulting expression is precisely the right-hand side of (3.42).

Let us now return to theorem 1. It turns out to be convenient to prove directly not (4.1), but rather the limit theorem

$$
\lim _{a \rightarrow \infty} E_{\beta}^{\mathrm{hard}}\left(Q_{a}(s) ; c a / 2\right)=F_{\beta}(s) \quad Q_{a}(s):=\left(a-\left(\frac{a}{2}\right)^{1 / 3} s\right)^{2} \quad(\beta=1,2) .
$$

Let us show that if we can prove (4.2) via proposition 2, and thus prove that

$$
\begin{aligned}
\lim _{a \rightarrow \infty} \int_{0}^{Q_{a}(s)} & \mathrm{d} x_{1} \cdots \int_{0}^{Q_{a}(s)} \mathrm{d} x_{k} \rho_{k}^{\text {hard }}\left(x_{1}, \ldots, x_{k}\right) \\
= & \int_{s}^{\infty} \mathrm{d} x_{1} \cdots \int_{s}^{\infty} \mathrm{d} x_{k} \rho_{k}^{\text {soft }}\left(x_{1}, \ldots, x_{k}\right)
\end{aligned}
$$

where

$$
\begin{aligned}
& \rho_{k}^{\text {hard }}\left(x_{1}, \ldots, x_{k}\right)=\operatorname{det}\left[K_{\beta}^{\text {Bessel }}\left(x_{j}, x_{l}\right)\right]_{j, l=1, \ldots, k} \\
& \rho_{k}^{\text {soft }}\left(x_{1}, \ldots, x_{k}\right)=\operatorname{det}\left[K_{\beta}^{\text {Airy }}\left(x_{j}, x_{l}\right)\right]_{j, l=1, \ldots, k}
\end{aligned}
$$

(the criterion (3.6) has already been checked for $\rho_{k}^{\text {soft }}$, so checking (4.3) is sufficient to deduce (4.2)), and further show that

$$
\lim _{a \rightarrow \infty} \prod_{l=1}^{k}\left(-Q_{a}^{\prime}\left(x_{l}\right)\right) \rho_{k}^{\text {hard }}\left(Q_{a}\left(x_{1}\right), \ldots, Q_{a}\left(x_{k}\right)\right)=\rho_{k}^{\text {soft }}\left(x_{1}, \ldots, x_{k}\right)
$$

with convergence uniform on compact sets, then (4.1) holds.

Lemma 1. Define $Q_{a}(s)$ as in (4.2), and suppose (4.6) and (4.3) hold. Then

$$
\begin{gathered}
\lim _{a \rightarrow \infty} \int_{0}^{A_{a}(s)+O(a)} \mathrm{d} x_{1} \cdots \int_{0}^{A_{a}(s)+O(a)} \mathrm{d} x_{k} \rho_{k}^{\text {hard }}\left(x_{1}, \ldots, x_{k}\right) \\
=\int_{s}^{\infty} \mathrm{d} x_{1} \cdots \int_{s}^{\infty} \mathrm{d} x_{k} \rho_{k}^{\text {soft }}\left(x_{1}, \ldots, x_{k}\right)
\end{gathered}
$$

where $A_{a}(s)=a^{2}-2 a(a / 2)^{1 / 3} s$, and consequently (4.1) holds. 
Proof. Now $Q_{a}(s)=A_{a}(s)+O\left(a^{2 / 3}\right)$, so the left-hand side of (4.7) is unchanged if we replace $A_{a}(s)$ by $Q_{a}(s)$. Doing this, then noting

$$
\begin{aligned}
\lim _{a \rightarrow \infty} \int_{0}^{Q_{a}(s)+O(a)} \mathrm{d} x_{1} \ldots \int_{0}^{Q_{a}(s)+O(a)} \mathrm{d} x_{k} \rho_{k}^{\text {hard }}\left(x_{1}, \ldots, x_{k}\right) \\
=\int_{s+O\left(a^{-1 / 3}\right)}^{2(a / 2)^{2 / 3}} \mathrm{~d} x_{1} \cdots \int_{s+O\left(a^{-1 / 3}\right)}^{2(a / 2)^{2 / 3}} \mathrm{~d} x_{k} \prod_{l=1}^{k}\left(-Q_{a}^{\prime}\left(x_{l}\right)\right) \rho_{k}^{\text {hard }}\left(Q_{a}\left(x_{1}\right), \ldots, Q_{a}\left(x_{k}\right)\right)
\end{aligned}
$$

it follows from (4.3) that (4.7) will hold provided

$$
\lim _{a \rightarrow \infty} \int_{s+O\left(a^{-1 / 3}\right)}^{s} \mathrm{~d} x_{1} \cdots \int_{s+O\left(a^{-1 / 3}\right)}^{s} \mathrm{~d} x_{k} \prod_{l=1}^{k}\left(-Q_{a}^{\prime}\left(x_{l}\right)\right) \rho_{k}^{\text {hard }}\left(Q_{a}\left(x_{1}\right), \ldots, Q_{a}\left(x_{k}\right)\right)=0 .
$$

But from (4.6) the integrand is bounded, so this holds true. With (4.7) established, (4.1) follows from proposition 2 .

To establish (4.6) and (4.3), we first prove the analogue of proposition 7.

Proposition 8. Let $Q_{a}(s)$ be given as in (4.2). For $\beta=1,2$, as $a \rightarrow \infty$

$$
\begin{array}{r}
\left(Q_{a}^{\prime}(x) Q_{a}^{\prime}(y)\right)^{1 / 2} K_{\beta}^{\mathrm{Bessel}}\left(Q_{a}(x), Q_{a}(y)\right)=K_{\beta}^{\text {Airy }}(x, y) \\
+O\left(\frac{1}{a^{1 / 3}}\right) \begin{cases}O\left(\mathrm{e}^{-x}\right) & \beta=1 \\
O\left(\mathrm{e}^{-x-y}\right) & \beta=2\end{cases}
\end{array}
$$

where the remainder terms hold uniformly for $x, y \in(s, \infty)$.

Proof. Consider first the case $\beta=2$. Recalling (3.11) and the definition of $Q_{a}$ from (4.2), we see asymptotic estimates of

$$
J_{a}\left(a-\left(\frac{a}{2}\right)^{1 / 3} s\right) \quad J_{a}^{\prime}\left(a-\left(\frac{a}{2}\right)^{1 / 3} s\right)
$$

are required. Now results of Olver [28, ch 11, sections 10.1-10.4] imply the uniform asymptotic expansion

$$
J_{v}(v z) \sim \frac{1}{v^{1 / 3}}\left(\frac{4 \zeta}{1-z^{2}}\right)^{1 / 4}\left\{\operatorname{Ai}\left(v^{2 / 3} \zeta\right)+O\left(v^{-4 / 3}\right) O\left(\mathrm{e}^{-v^{2 / 3} \zeta}\right)\right\}
$$

valid for all $z \in \mathbb{C}, \arg z \neq \pi$, where $\zeta=\zeta(z)$ is specified by

$$
\frac{2}{3} \zeta^{3 / 2}=\log \frac{1+\left(1-z^{2}\right)^{1 / 2}}{z}-\left(1-z^{2}\right)^{1 / 2}
$$

Thus for $z \rightarrow 0^{+}, \zeta$ diverges to $+\infty$, then monotonically decreases to 0 at $z=1$, where it has the power series expansion

$$
\zeta(z)=2^{1 / 3}(1-z)+O\left((1-z)^{2}\right)
$$

We remark that the term $O\left(\mathrm{e}^{-v^{2 / 3} \zeta}\right)$ in (4.9) can be strengthened to involve the exponent $\left(v^{2 / 3} \zeta\right)^{3 / 2}$, but this refinement is not needed for our purpose. Let us set $z=1-2^{-1 / 3} w / v^{2 / 3}$, where $0<w<2^{1 / 3} v^{2 / 3}$ and thus $0<z<1$. Making use of (4.9), (4.10) and the fact that $\zeta(1-z)$ is an increasing function for $0<z<1$, and using Taylor's theorem to estimate $\operatorname{Ai}(w+\epsilon),|\epsilon| \ll 1$, shows

$$
J_{v}\left(v-w(v / 2)^{1 / 3}\right) \sim\left(\frac{2}{v}\right)^{1 / 3} \operatorname{Ai}(w)+O\left(\frac{1}{v}\right) O\left(\mathrm{e}^{-w}\right) .
$$


To obtain the analogous expansion of $J_{v}^{\prime}$, we make use of the formula

$$
J_{v}^{\prime}(z)=\frac{1}{2}\left(J_{v-1}(z)-J_{v+1}(z)\right) .
$$

Setting $v \mapsto v \pm 1$, then $z=\frac{v}{v \pm 1}\left(1-w / 2^{1 / 3} v^{2 / 3}\right)$ in (4.9) shows

$$
\begin{aligned}
J_{v \pm 1}\left(v-w(v / 2)^{1 / 3}\right) & \sim\left(\frac{2}{v}\right)^{1 / 3} \operatorname{Ai}\left(w \pm \frac{2}{v^{1 / 3}}\right)+O\left(\frac{1}{v}\right) O\left(\mathrm{e}^{-w}\right) \\
& \sim\left(\frac{2}{v}\right)^{1 / 3} \operatorname{Ai}(w) \pm\left(\frac{2}{v}\right)^{2 / 3} \operatorname{Ai}^{\prime}(w)+O\left(\frac{1}{v}\right) O\left(\mathrm{e}^{-w}\right)
\end{aligned}
$$

where to obtain the second line the Airy function has been expanded to first order, and the error estimated using Taylor's theorem. Substituting this in (4.12) shows that apart from the exponent in the term $O\left(v^{-1}\right),(4.11)$ in fact remains valid upon formal differentiation with respect to $w$, and thus

$$
-\left(\frac{v}{2}\right)^{1 / 3} J_{v}^{\prime}\left(v-w(v / 2)^{1 / 3}\right) \sim\left(\frac{2}{v}\right)^{1 / 3} \mathrm{Ai}^{\prime}(w)+O\left(v^{-2 / 3}\right) O\left(\mathrm{e}^{-w}\right) .
$$

Making use of (4.11) and (4.13) in (3.11) with the substitution $x \mapsto Q_{a}(x), y \mapsto Q_{a}(y)$, gives (4.8) in the case $\beta=2$, provided $|x-y|$ is bounded away from zero. This latter proviso is needed at this stage due to the term $x-y$ in the denominator of the definition of $K_{2}^{\text {hard }}$, which could affect the decay of the error term. To see that in fact no such complication arises, for $|x-y| \ll 1$, we make use of $K_{2}^{\text {hard }}$ being an analytic function in both $x$ and $y$, and so permitting the Cauchy-type integral representation

$$
K_{2}^{\text {hard }}(x, y)=\frac{1}{2 \pi} \int_{0}^{2 \pi} K_{2}^{\text {hard }}\left(x, y+R \mathrm{e}^{\mathrm{i} t}\right) \mathrm{d} t
$$

for arbitrary $R>0$. Choosing $R=2 a(a / 2)^{1 / 3}$ we see that to use this formula to analyse the left-hand side of (4.8) we require the asymptotic estimates (4.11) and (4.13) with $w=y-\mathrm{e}^{\mathrm{i} t}+O\left(v^{-2 / 3}\right)$. Note that the modulus of the error terms therein is $O\left(\mathrm{e}^{-y}\right)$. Substituting in (4.14) gives the Cauchy-type integral representation of $K_{2}^{\text {soft }}(x, y)$ as the leading term while the remainder term is seen to be bounded by terms $O\left(a^{-1 / 3}\right) O\left(\mathrm{e}^{-x-y}\right)$ coming from the numerator in the definition of $K_{2}^{\text {hard }}\left(x, y+R \mathrm{e}^{\mathrm{i} t}\right)$, times the maximum of the scaled denominator

$$
\frac{2 a(a / 2)^{1 / 3}}{\left|Q_{a}(x)-Q_{a}(y)+R \mathrm{e}^{\mathrm{i} t}\right|} \sim \frac{1}{\left|y-x+\mathrm{e}^{\mathrm{i} t}\right|} .
$$

Because this is bounded for $|x-y| \ll 1$, we see that the error term in (4.14) is indeed as stated in (4.8) for $\beta=2$.

From the definition (3.25), to derive (4.8) in the case $\beta=1$ the only remaining task is to give the asymptotic expansion of

$$
\int_{0}^{Q_{a}(y)} J_{a}(t) \mathrm{d} t=-\int_{s}^{2(a / 2)^{2 / 3}} Q_{a}^{\prime}(t) J_{a}\left(Q_{a}(t)\right) \mathrm{d} t .
$$

But this follows immediately from (4.11), giving the form required by (4.8).

With the asymptotic formulae (4.8) substituted in (4.4), we see that

$$
\prod_{l=1}^{k}\left(-Q_{a}^{\prime}\left(x_{l}\right)\right) \rho_{k}^{\text {hard }}\left(Q_{a}\left(x_{1}\right), \ldots, Q_{a}\left(x_{k}\right)\right)=\rho_{k}^{\text {soft }}\left(x_{1}, \ldots, x_{k}\right)+O\left(a^{-1 / 3}\right) O\left(\mathrm{e}^{-x_{1}-\cdots-x_{k}}\right) .
$$

This shows immediately that (4.6) and (4.3) hold. Consequently the limit formula (4.2) is proved, and thus, via lemma 1 , so is (4.1). 
To use (4.1), and its extension to the case $\beta=4$ noted in corollary 2, to deduce the scaled limits of the probabilities $\operatorname{Pr}\left(L^{\square}(t) \leqslant l\right), \operatorname{Pr}\left(L^{\square}(t) \leqslant 2 l\right)$ and $\operatorname{Pr}\left(L^{\square}(t) \leqslant 2 l\right)$ we see from proposition 1 that we should rewrite the former so that it specifies $\lim _{t \rightarrow \infty} E_{\beta}^{\text {hard }}(4 t ; f(t))$ with $f(t)$ a positive integer (even positive integer in the case $\beta=4$ ). For this purpose we set $a=2\left[t^{1 / 2}+\frac{1}{2} t^{1 / 6} s\right]$ where [ ] denotes the integer part, and make an appropriate choice of the arbitrary term $O(a)$ so that (4.1) reads

$$
\lim _{t \rightarrow \infty} E_{\beta}^{\text {hard }}\left(4 t ; c\left[t^{1 / 2}+\frac{1}{2} t^{1 / 6} s\right]\right)=F_{\beta}(s)
$$

where $c=1$ for $\beta=1$ and $c=2$ for $\beta=2$, 4. Setting $l=c\left[t^{1 / 2}+\frac{1}{2} t^{1 / 6} s\right]$ in the formulae of proposition 1, we then reclaim from (4.15) the limit formulae (1.4), (1.5) and (1.7), equivalent upon de-Poissonization to limit theorems of Baik, Deift and Johansson [3], and Baik and Rains [5].

\section{Acknowledgments}

This research was partially conducted during the period AB served as a Clay Mathematics Institute Long-Term Prize Fellow, and was supported in part by the NSF grant DMS-9729992. The work of PJF was supported by the Australian Research Council. Thanks are due to Eric Rains for pointing out the significance of the zonal polynomial identities (2.19)-(2.21), and to Percy Deift for facilitating this collaboration by supporting a visit of PJF to the University of Pennsylvania during April 2001. Helpful comments of a referee are also acknowledged.

\section{References}

[1] Aldous D and Hammersley P D 1995 Interacting particle process and longest increasing subsequences Probab. Theory Rel. Fields 103 199-213

[2] Aldous D and Diaconis P 1999 Longest increasing subsequences: from patience sorting to the Baik-DeiftJohansson theorem Bull. Am. Math. Soc. 36 413-32

[3] Baik J, Dieft P and Johansson K 1999 On the distribution of the length of the longest increasing subsequence of random permutations J. Am. Math. Soc. 12 1119-78

[4] Baik J and Rains E M 2001 Algebraic aspects of increasing subsequences Duke Math. J. 109 1-65

[5] Baik J and Rains E M 2001 The asymptotics of monotone subsequences of involutions. Duke Math. J. 109 205-81

[6] Borodin A 1999 Biorthogonal ensembles Nucl. Phys. B 536 704-32

[7] Borodin A, Okounkov A and Olshanski G 2000 On asymptotics of Plancherel measures for symmetric groups J. Am. Math. Soc. 13 481-515

[8] Dueñez E 2001 Random matrix ensembles associated to compact symmetric spaces Preprint math-ph/0111005

[9] Dumitriu I and Edleman A 2002 Matrix models for beta ensembles J. Math. Phys. 43 5830-47

[10] Forrester P J 1993 Exact results and universal asymptotics in the Laguerre random matrix ensemble J. Math. Phys. 35 2539-51

[11] Forrester P J 1993 The spectrum edge of random matrix ensembles Nucl. Phys. B 402 709-28

[12] Forrester P J 2000 Painlevé transcendent evaluation of the scaled distribution of the smallest eigenvalue in the Laguerre orthogonal and symplectic ensembles Preprint nlin.SI/0005064

[13] Forrester P J Log-gases and random matrices webpage www.ms.unimelb.edu.au/ matpjf/matpjf.html.

[14] Forrester P J and Hughes T D 1994 Complex Wishart matrices and conductance in mesoscopic systems: exact results J. Math. Phys. 35 6736-47

[15] Forrester P J, Nagao T and Honner G 1999 Correlations for the orthogonal-unitary and symplectic-unitary transitions at the hard and soft edges Nucl. Phys. B 553 601-43

[16] Forrester P J and Rains E M 2002 Correlations for superpositions and decimations of Laguerre and Jacobi orthogonal matrix ensembles with a parameter Preprint math-ph/0211041

[17] Forrester P J and Witte N S 2002 Application of the $\tau$-function theory of Painlevé equations to random matrices: PVI, the JUE,CyUE, cJUE and scaled limits Preprint math-ph/0204008

[18] James A T 1964 Distribution of matrix variates and latent roots derived from normal samples Ann. Math. Stat. 35 475-501 
[19] Johansson K 1998 The longest increasing subsequence in a random permutation and a unitary random matrix model Math. Res. Lett. 5 63-82

[20] Johansson K 2000 Shape fluctuations and random matrices Commun. Math. Phys. 209 437-76

[21] Johansson K 2001 Discrete orthogonal polynomial ensembles and the Plancherel measure Ann. Math. 153 259-96

[22] Johnstone I M 2001 On the distribution of the largest eigenvalue in principal components analysis Ann. Stat. 29 295-327

[23] Kaneko J 1993 Selberg integrals and hypergeometric functions associated with Jack polynomials SIAM J. Math Anal. 24 1086-110

[24] Lenard A 1973 Correlation functions and the uniqueness of the state in classical statitical mechanics Commun. Math. Phys. 30 35-44

[25] Macdonald I G 1995 Hall Polynomials and Symmetric Functions 2nd edn (Oxford: Oxford University Press)

[26] Muirhead R J 1982 Aspects of Multivariable Statistical Theory (New York: Wiley)

[27] Okounkov A 2000 Random matrices and random permutations Int. Math. Res. Notices 20 1043-95

[28] Olver F W J 1974 Asymptotics and Special Functions (New York: Academic)

[29] Rains E M 1995 Attack of the zonal polynomials Preprint

[30] Rains E M 1998 Increasing subsequences and the classical groups Elect. J. Combinat. 5 \#R12

[31] Rains E M $2001 B C_{n}$-symmetric polynomials Preprint math.QA/0112035

[32] Soshnikov A 2000 Determinantal random point fields Russian Math. Surveys 55 923-75

[33] Soshnikov A 2001 A note on the universality of the distribution of the largest eigenvalues in certain sample covariance matrices Preprint math.PR/0104113

[34] Stanley R P 1989 Some combinatorial properties of Jack symmetric functions Adv. Math. 77 76-115

[35] Tracy C A and Widom H 1994 Level-spacing distributions and the Airy kernel Commun. Math. Phys. 159 $151-74$

[36] Tracy C A and Widom H 1994 Level-spacing distributions and the Bessel kernel Commun. Math. Phys. 161 289-309

[37] Tracy C A and Widom H 1996 On orthogonal and symplectic matrix ensembles Commun. Math. Phys. 177 727-54

[38] Whittaker E T and Watson G N 1965 A Course of Modern Analysis 2nd edn (Cambridge: Cambridge University Press) 